Revue d'histoire de l'Amérique française

REVUE D.HISTOIRE DE L'AMÉRIQUE FRANÇAISE

\title{
Saint Antoine Daniel, martyr canadien (suite)
}

\section{Fernand Potvin}

Volume 8, numéro 4, mars 1955

URI : https://id.erudit.org/iderudit/301679ar

DOI : https://doi.org/10.7202/301679ar

Aller au sommaire du numéro

Éditeur(s)

Institut d'histoire de l'Amérique française

ISSN

0035-2357 (imprimé)

1492-1383 (numérique)

Découvrir la revue

Citer cet article

Potvin, F. (1955). Saint Antoine Daniel, martyr canadien (suite). Revue d'histoire de l'Amérique française, 8(4), 556-564. https://doi.org/10.7202/301679ar d'utilisation que vous pouvez consulter en ligne.

https://apropos.erudit.org/fr/usagers/politique-dutilisation/ 


\title{
SAINT ANTOINE DANIEL, MARTYR CANADIEN * (suite)
}

\section{Chapitre II}

\author{
DEUX ANS D'ATTENTE : LE CAP-BRETON ET QUÉBEC \\ $(1632-34)$
}
Rétrocession du Canada à la France - Retour des Jésuites - Départ du PÈre Daniel pour le Cap-Breton - Arrivée $\lambda$ Québec - Tentative d'embarquement pour la Huronie - SÉJOUR DANS LA CAPITALE.

La colonie canadienne, fondée par Champlain en 1608, ne s'était développée que lentement, malgré l'énergie de son chef et de ses premiers "habitants". Grâce à la paix, toutefois, sa condition, au cours des vingt années qui suivirent, allait chaque jour s'améliorant davantage lorsque arriva de Tadoussac, le 10 juillet 1628, la lettre des frères Kirke réclamant l'habitation de Québec.'

La réponse de Champlain à leur ultimatum fut si fière et si courageuse que les Kirke n'osèrent pas se présenter devant la ville avant le printemps suivant. Il leur suffisait, d'ailleurs, d'intercepter à Tadoussac les convois de secours et de réduire ainsi la colonie à la famine. ${ }^{2}$ Ce plan ne réussit que trop bien : après quelques mois d'un siège héroïque, Champlain dut enfin reconnaître l'inutilité de sa résistance. Il remit Québec aux Anglais et repassa en France. ${ }^{3}$

*Voir, Revue d'Histoire de l'Amérique française (décembre 1954): 395-414.

1. "Messieurs, ie uous aduise comme i'ay obtenu Commission du Roy de la grande Bretagne, mon tres-honoré Seigneur \& Maistre, de prendre possession de ces paîs, sçacvoir Canadas \& l'Acadie... David Quer. Du bord de la Vicaille ce 18. Iuillet 1628. Stille vieux", Champlain, 5: 279-282.

2. "... car ie sçay que quand vous serez incommodé de uiures, i'obtiendray plus facillement ce que ie desire, qui est d'auoir l'habitation \& pour empescher que nul navire ne uiene, ie resous de demeurer icy...," ibid., 5 : 280.

3. Ibid., $6: 143$ ss. 
$\AA$ son arrivée en Europe, il apprit que la paix entre la France et l'Angleterre avait été conclue à Suze dès le 24 avril 1629. Or il avait tenu Québec jusqu'au 19 juillet : la reddition ne pouvait donc pas avoir été valide puisqu'à ce moment l'état de guerre n'existait plus entre l'Angleterre et la France. Champlain insista auprès de Louis XIII et, à la suite de longues négociations entre le souverain français et Charles Ier, roi d'Angleterre, ce dernier signa, le 29 mars 1632, le traité de SaintGermain-en-Laye qui remettait à la France tous les postes occupés par les Anglais au Canada. ${ }^{4}$

Richelieu, qui éprouvait une grande sympathie pour les Capucins, leur offrit alors la Mission du Canada, mais, par déférence pour les anciens missionnaires qui s'étaient dépensés dans la colonie avant 1629, ils ne voulurent pas répondre à cette demande. Ainsi, le cardinal eut à choisir entre les Récollets et les Jésuites, car son expérience lui conseillait de n'envoyer d'abord qu'un seul ordre religieux dans cette nouvelle mission. Enfin, il se décida à faire appel aux Jésuites, estimant que la pauvreté très rigoureuse des Récollets les rendrait trop à charge à la colonie naissante. ${ }^{5}$

De leur côté, les Jésuites se tenaient prêts à partir. Dès le 6 décembre 1631, le Père Charles Lalemant, ancien supérieur de la Mission canadienne, avait écrit à l'assistant de la province de France à Rome afin de promouvoir le retour des missionnaires au Canada. ${ }^{6}$ Le Père Lalemant avait vu juste. Le traité de Saint-Germain-en-Laye était à peine signé que déjà le sieur Emery de Caen, commandant provisoire de la colonie, appareillait pour la Nouvelle-France. Sur l'un des vaisseaux avaient pris place le Père Paul Le Jeune, nouveau Supérieur de la Mission, le Père Anne de Nouë et le frère coadjuteur Gilbert Burel.'

4. "De la part de Sa Majesté de la Grande Bretagne, le dit Sieur Ambassadeur... a promis et promet, pour et au nom de sa dite Majesté, de rendre et de restituer à Sa Majesté Très Chrétienne, tous les lieulx occupez en la Nouvelle-France, la Cadie et Canada par les subjets de Sa Majesté de la Grande-Bretagne, y ceulx faire retirer des dits lieulx", Traité de paix entre la France et l'Angleterre, Saint-Germain-en-Laye, le 29 mars, 1631, Collection des manuscrits, 1: 88.

5. Rochemonteix, 1: 182; cette décision suscita malheureusement de longues querelles entre les deux ordres religieux, voir ibid., 1 : 184, note $2,186-87$.

6. Voir ch. I, 19.

7. Ils quittèrent Honfleur le 18 avril 1632 pour n'arriver à Québec que le 5 juillet. Voir Q 1632, $1 \mathrm{~g}$ et 7d; Rochemonteix, $1: 186$. 
Peu après le départ d'Emery de Caen, le capitaine Charles Daniel, comme l'avait annoncé le Père Lalemant, s'embarqua probablement pour le fort Sainte-Anne qu'il avait construit à l'embouchure de la rivière Grand-Cibou, lors de sa fameuse expédition de 1629 au Cap-Breton. ${ }^{8}$ En même temps et peut-être sur le même navire, ${ }^{9}$ traversaient son frère Antoine et le Père Ambroise Davost. ${ }^{10}$ Ainsi se réalisait après plusieurs années d'attente, le désir d'apostolat que le Père Daniel avait sans doute ressenti dès son séjour au collège de Rouen alors que, tout jeune religieux encore, il avait rencontré le petit Louis Amantacha que le Père Lalemant voulait faire instruire en France."'

Vers les premiers jours de l'été 1632, le Père Daniel aperçut enfin les rives du Cap-Breton et, bientôt après, il put distinguer une petite habitation: c'était le fort Sainte-Anne qui protégeait l'entrée de la rivière Grand-Cibou. Leur arrivée causa une joie très vive à tous ces soldats, isolés depuis un an dans un pays désert et inhospitalier. ${ }^{2}$ Mais qui pourrait dire le bonheur avec lequel le commandant du fort accueillit ses deux frères, le capitaine Charles et surtout le Père Antoine qu'il n'avait pas revus depuis un an au moins ? $\AA$ la nouvelle que les Pères allaient demeurer avec eux, les Français éprouvèrent un profond soulagement car, depuis le départ des Pères Vimont et de Vieuxpont, ils n'avaient pu bénéficier des secours de la religion. Pendant quelques jours, on procéda au déchargement des fournitures nécessaires à l'habitation et à la traite des fourrures avec les Indiens. Puis, le capitaine Daniel, après avoir pourvu au bon ordre de son établissement, mit à la voile pour retourner en France.

Le Père Daniel et le Père Davost ne furent pas longtemps au fort Sainte-Anne sans ressentir amèrement la solitude immense qui les en. tourait. Impression pénible, certes, pour ces coeurs d'apôtres qui n'avaient

8. Voir ch. I, 9-10.

9. "It is not definitely known how long the garrison was kept there : but, when de Caen took possession of Canada (1632), the Jesuits Davost and A. Daniel also came to cape Breton (probably with Charles, the latter's brother), and carried on the mission begun by Vimont", JR $4: 269-70$, note 46 . Voir ch. I, 12, note 28 .

10. "En même temps, le Père Antoine Daniel s'établissait avec le Père Davost au Cap-Breton, où commandait son frère, le capitaine Charles Daniel, et où l'on désirait vivement le retour des missionnaires", Rochemonteix, $1: 181$.

11. Voir ch. I, 15-16.

12. “... ces endroits ne sont pas beaucoup plaisans ni agreables que pour la pesche de moluë", Champlain, 6:181. 
traversé l'océan que dans l'espoir de s'employer corps et âme à la conversion des sauvages. Déjà leurs prédécesseurs avaient rapporté à Champlain que "durant l'hyvernement, ils virent peu de sauuages qui n'y viennent au fort que par rencontre chercher les vaisseaux François qui y peuuent estre pour traitter auec eux". ${ }^{13}$ Le Père Daniel s'employa de son mieux cependant, à secourir de son ministère les soldats qui gardaient le fort, et, chaque fois que s'en présentait l'occasion, il essayait d'amorcer quelques contacts avec les Indiens de l'endroit. Ainsi s'écoulèrent les premiers mois de son apostolat en terre canadienne.

L'année suivante, Champlain revint au Canada pour reprendre définitivement en main la colonie qu'il avait fondée. Son navire amenait en même temps deux nouveaux missionnaires ou plutôt deux vétérans : les Pères Jean de Brébeuf et Ennemond Massé. Le 5 mai, cette année-là jour de l'Ascension, ils abordèrent à la rivière Grand-Cibou, face à l'habitation du capitaine Daniel. Leur venue apporta beaucoup de joie aux Pères Daniel et Davost qui avaient passé un dur hiver dans le petit poste isolé. ${ }^{14}$ Le Père Massé leur communiqua alors l'ordre du Père Le Jeune qui les invitait à se rendre à Québec. $\bar{A}$ cette nouvelle, Champlain $s^{\prime}$ empressa de leur offrir passage sur son navire, ce que les Pères Daniel et Davost acceptèrent volontiers. Quelques jours plus tard, ils s'embarquaient avec lui pour Québec. ${ }^{15}$

Le 22 mai suivant, le Père de Brébeuf arrivait à destination. ${ }^{16}$ Pour ajouter encore une heureuse nouvelle à la joie générale que causait son retour, il annonça aux Pères Le Jeune et de Nouë "que le pere Masse estoit à Tadoussac; que le Père Daniel, et le Pere Dauost nous venoient voir du grand Chibou"." Le Père Daniel demeura quelque temps à

13. Champlain, 6: 181-2; "Quod ad Barbaros attinet, qui in illis locis rari usnt et infrequentes...", Monumenta bistorica missionis Canadensis, Cap. VIII, texte cité par Rochemonteix, 1: 182, note de la page précédente.

14. "On Ascension Thursday, May 5, they [FF. de Brébeuf and Massé] anchored before Daniel's Fort on Cape Breton Island. There they found Antoine Daniel and Ambroise Davost, who had passed a wretched winter and discovered few natives to convert. Massé ordered the two Jesuits to proceed to Quebec", F. X. Talbot, Saint among the Hurons (New York, 1949), 95.

15. Ibid., 95; L. Le Jeune, Dictionnaire général, $1: 470 \mathrm{~g}$.

16. P. Le Jeune, Q 1633, 25d.

17. Ibid., $26 \mathrm{~g}$. 
Tadoussac malgré son impatience d'arriver à Québec. Comme le retard menaçait de se prolonger, il décida de se rendre à destination "par le moyen d'vne barque qui montoit à Kebec". ${ }^{18}$ Quelques jours plus tard, soit le 3 juillet, le Père Davost arriva aussi de Tadoussac. Il avait de même fait le voyage en canot, ne voulant pas attendre plus longtemps le vent favorable qui permettrait aux navires de lever l'ancre. Comme le Père Daniel, il désirait se rendre le plus tôt possible à Québec, "craignant d'ailleurs que les Hurons ne descendissent, et ne s'en retournassent sans luy en leur pays. Dieu soit Glorifié pour iamais, qui nous a rassemblez tous en nostre petite maisonnette, auec vne grande ioye et vn grand desir de luy offrir nos vies pour son service". 19 Ainsi se trouvaient réunis à Québec, un an à peine après la restitution du Canada à la France, les six premiers missionnaires qui allaient entreprendre pour de bon la conquête spirituelle de ce nouveau royaume.

Les Pères de Brébeuf, Daniel et Davost étaient donc arrivés en Nouvelle-France dans le but de se rendre le plus tôt possible en Huronie. Seul le Père de Brébeuf avait quelque connaissance de la langue huronne, mais le Père Le Jeune croyait qu'on ne devait pas remettre à plus tard l'évangélisation de ce peuple. Il comptait, d'ailleurs, que l'apprentissage de la langue se ferait encore plus rapidement au milieu des Hurons. Lui-même devait entreprendre, l'hiver suivant, une terrible expédition de chasse avec les Montagnais dans le seul but de perfectionner sa connaissance de la langue. ${ }^{20}$

Cette année-là, les Hurons descendirent en grand nombre vers les Français ${ }^{21}$ et, grâce aux pressantes invitations de Louis Amantacha, ils se rendirent presque tous à Québec où les attendaient avec impatience les trois Pères qui se proposaient de retourner avec eux. Le lendemain de leur arrivée, soit le 29 juillet, se tint un grand conseil où les chefs

18. Ibid., 30g.

19. Ibid., $31 \mathrm{~g}$.

20. "... il ne sera plus de besoin de faire ces courses, quand on aura la cognoissance des langues, et qu'on les aura réduites en préceptes", $P$. Le Jeune, $Q 1634,87 d$.

21. "... il en estoit desia venu quelques canots en diuers iours, tantost sept ou huit, et tantost dix ou douze à la fois, mais en fin le 28. de Iuillet il en est arriué cent quarante ou enuiron tout à la fois, qui portoient bien cinq cens Hurons, d'autres disent 700, auec leurs marchandises", $P$. Le Jeune, $Q 1633,34 \mathrm{~g}$. 
hurons parlèrent avec éloquence de l'amitié qui les unissait aux Français; en témoignage de quoi ils offrirent de nombreux présents à Champlain. Celui-ci se leva à son tour et, avec beaucoup d'adresse et de circonspection, les informa que des Robes Noires désiraient se rendre en leur pays, ce dont les sauvages se montrèrent fort satisfaits. Bref, ce jour-là et les suivants, les Hurons semblaient parfaitement disposés à recevoir les trois Pères et les quelques domestiques: Champlain n'avait pas ménagé les présents mais l'enjeu en valait le prix. ${ }^{22}$

Le 4 août, les Pères remirent aux Hurons le petit bagage qu'ils avaient préparé déjà depuis longtemps; le départ avait été fixé pour le lendemain matin. Un incident fâcheux fit cependant échouer toute l'entreprise. Les Français détenaient un Algonquin qui avait été convaincu du meurtre d'un des leurs. Malgré les instances des Hurons, Champlain ne permit pas son élargissement. Or, "sur les dix ou onze heures du soir, vn Sauuage borgne de la nation de l'Isle grandement allié de la nation du prisonnier, s'en alla crier par les cabanes de tous les Sauuages, qu'on se donnast bien garde d'embarquer aucun François et que les parens du prisonnier estoyent aux aguets sur la riuière pour tuer les François s'ils les pouuoyent attrapper au passage". ${ }^{23}$ Cette rumeur changea complètement les dispositions des Hurons et malgré tous les arguments que Champlain put leur apporter, ils demeurèrent inflexibles. ${ }^{24}$

Les Pères de Brébeuf, Daniel et Davost en furent quittes pour leurs préparatifs car, selon l'avis de Champlain, ${ }^{25}$ il fut reconnu plus prudent et plus avantageux de retarder leur départ à l'année suivante que de risquer une guerre entre des Indiens alliés, guerre où les Français se trouveraient forcément impliqués. Mais déjà les missionnaires avaient manifesté clairement quel était leur courage, car le Père Le Jeune n'hésite pas à leur rendre ce magnifique témoignage: "Le Pere Brebeuf voiant que son voyage estoit rompu, et que ce seroit temerité de l'entreprendre, non pour la crainte de la mort, car ie ne les vy iamais si resolus,

22. Ibid., 34-40g.

23. P. Le Jeune, $Q 1633,40 \mathrm{~g}$.

24. "Le sieur de Champlain voyant ce changement si subit, fit tout ce qu'il peut, et nous donna liberté de proposer toutes les raisons que nous pourrions pour faire en sorte que nos peres se peussent mettre en chemin. Il apporta des raisons tres-fortes, et tres-pertinentes; il se seruit de menace; il leur proposa la paix, et la guerre : bref on ne pouuoit rien souhaitter dauantage", P. Le Jeune, ibid., 41g.

25. Ibid., $42 \mathrm{~g}$. 
luy et ses deux compagnons le Pere Daniel et le Pere Dauost, qu'alors qu'on parla qu'ils pourroient laisser la vie au chemin qu'ils entreprenoient pour la gloire de nostre Seigneur". ${ }^{26}$

Les Hurons mirent la dernière main à leurs préparatifs et se montrèrent fort désolés de ce contretemps qui empêchait les Pères de les accompagner. Le lendemain, ils quittèrent Québec pour "les pays d'en haut", laissant derrière eux les missionnaires qui n'étaient partis de France que pour venir partager leur vie. Ce fut une douloureuse déception pour le Père Daniel. Dans la Relation de 1633, le Père Le Jeune écrit : "Si nos Peres fussent entrés cette année aux Hurons, ie m'attendois de rescrire à V. R. l'an prochain que, receperat Samaria verbum Dei, que ces barbares auoient receu la foy; ce sera quand il plaira à celuy duquel dépend ce grand ouurage : car à mon aduis les hommes y peuuent bien peu, quoy qu'ils n'y doiuent espargner ny leurs trauaux, ny leur sang, ny leur vie". ${ }^{27}$

Puisque la Huronie leur était fermée pour cette année, les missionnaires acceptèrent de se livrer, avec autant d'ardeur, au travail qui était pour eux le plus urgent et le plus nécessaire: l'étude des langues indiennes. Le Père Le Jeune achève à peine le récit de leur départ manqué que déjà il annonce: "Nos Peres ne seront point icy oisifs. Le Pere Brebeuf leur fera leçon, tous les iours soirs et matins, de la langue des Hurons"; ${ }^{28}$ plus loin, il écrit encore: "Ie voy vn grand desir en nos Peres de deuorer toutes ces difficultez qui se rencontrent dans l'estude de ces langues, et vous diriez quasi que Dieu les a arrestez pour les acquerir icy plus commodement, afin qu'ils puissent à mesme temps mettre le feu en diuers endroits des Hurons quand sa Maiesté leur y donnera entrée". ${ }^{29}$ Tout leur apostolat, en effet, dépendait de leur habileté à se faire bien entendre, de sorte que l'établissement et le progrès de la religion seraient, pour une grande part, le résultat direct de leur application à cette étude. On peut deviner alors l'acharnement avec lequel le Père Daniel dut s'employer à cette tâche qui, certes, n'était pas facile; pour

26. P. Le Jeune, Q 1633, 41d-42g.

27. Ibid., $43 \mathrm{~d}$.

28. Ibid., $43 \mathrm{~g}$

29. P. Le Jeune, Q 1633, 43d. 
tout instrument de travail, ils ne disposaient que des connaissances que le Père de Brébeuf avait pu retenir de son premier séjour en Huronie, quatre années plus tôt.

Champlain, dès son retour à Québec en 1633, avait fait ériger la petite chapelle de Notre-Dame-de-la-Recouvrance pour remercier la Vierge d'avoir obtenu le retour du Canada aux Français. ${ }^{30}$ Le Père Daniel, outre l'étude de la langue huronne, desservit sans doute, ${ }^{31}$ comme les autres Pères, la petite chapelle que les premiers habitants, à l'exemple de leur gouverneur, ${ }^{32}$ fréquentaient régulièrement. Ainsi s'écoula, bien lentement au gré des missionnaires, le long hiver de 1633-1634; mais ils n'étaient pas hommes à abandonner la partie. Leur constance et leur application furent remarquables, comme en témoigne le Père Paul Le Jeune dans une lettre adressée à son Provincial, en 1634: "Nous avons vescu dans une grande paix, Dieu mercy, entre nous, avec nos gens, et avec tous nos françois. Je suis grandement édifié de tous nos Pères... Le P. Daniel et le P. Davost sont paisibles. Ils ont bien estudié à la langue huronne; j'ay tenu la main qu'ils ne fussent point divertis de cet exercice que ie croy estre de tres grande importance". ${ }^{33}$

Deux années s'étaient écoulées déjà depuis que le Père Daniel était arrivé en Nouvelle-France. Sa grande ambition était d'y travailler à la conversion des infidèles en leur prêchant la parole de Dieu, en leur donnant l'exemple d'une vie totalement consacrée au salut de leurs âmes. Mais qu'est-ce que la réalité lui avait apporté ? Un séjour temporaire au Cap-Breton, isolé au milieu de quelques soldats chrétiens, un faux départ pour la Huronie, puis, une autre année d'attente à Québec, vouée à l'étude de la langue et au ministère paroissial chez les colons français. Pour une âme d'apôtre, il n'y avait rien là de très réconfortant. La grande aventure missionnaire, dont il avait probablement rêvé avec beaucoup d'enthousiasme, débutait de façon fort prosaĩque. Ainsi le Père Daniei

30. Rochemonteix, 1: 200.

31. L. Cadieux et A. Pouliot, Gloires ontariennes II. Saint Antoine Daniel (Sudbury, 1948), 11; L.-M. Carles, Les Dieppois dans l'épopée canadienne, XVIle et XVIIIe siècles (Rouen, s.d.), 93.

32. P. Le Jeune, $Q 1634,2 \mathrm{~g}$.

33. P. Le Jeune au R. P. Provincial, 1634, Carayon, 123-24. 
commençait à réaliser le caractère non pas héroïque encore mais simplement austère de sa vocation. Au surplus, ce retard lui avait apporté une occasion providentielle de s'entraîner peu à peu à la rude vie des missionnaires et de s'exercer surtout à l'inépuisable patience que le travail d'évangélisation allait exiger.

Mais son "noviciat" pour la Huronie allait s'achever bientôt. Le Père Daniel était maintenant prêt à partir pour les "pays d'en haut", le corps et l'âme trempés d'une force nouvelle.

Fernand Porvin, s.j.b.a.

(à suivre)

AVIS - LA REVUE D'HISTOIRE DE L'AMERIQUE FRANÇAISE vit de ses abonnements, et rien que de ses abonnements. Elle compte que vous renouvellerez le vôtre, dès ces jours-ci, pour ne pas manquer le no de juin prochain, 1er de la 9ème année. 